\title{
Exoplanet detection capability of the COROT space mission
}

\author{
P. Bordé ${ }^{1}$, D. Rouan ${ }^{1}$, and A. Léger ${ }^{2}$ \\ 1 LESIA, UMR8109, Observatoire de Paris, 5 place Jules Janssen, 92195 Meudon, France \\ e-mail: Daniel.Rouan@obspm.fr \\ 2 Institut d'Astrophysique Spatiale, UMR 8617, Université Paris XI, 91405 Orsay Cedex, France \\ e-mail: Alain.Leger@ias.fr
}

Received 18 July 2003 / Accepted 25 April 2003

\begin{abstract}
COROT will be the first high precision photometric satellite to be launched with the aim of detecting exoplanets by the transit method. In this paper, we present the simulations we have carried out in order to assess the detection capability of COROT. Using the model of stellar population synthesis of the Galaxy developed at Besançon Observatory (Robin \& Crézé 1986) and a simple cross-correlation technique (Bordé et al. 2001), we find that COROT has the capacity to detect numerous exoplanets, not only Jupiter and Uranus-class ones, but also hot terrestrial planets, if they exist. We show that small exoplanets should be mainly gathered around 14-15th magnitude K2-M2 dwarfs and giant exoplanets around 15-16th magnitude F7-G2 dwarfs. We study the effect of crowding and the impact of a high stellar variability noise that both reduce the detection capability of the instrument.
\end{abstract}

Key words. stars: planetary systems - methods: statistical - techniques: photometric

\section{Introduction}

To date about a hundred exoplanets have been discovered spectroscopically by measuring the reflex motion of the star due to the gravitational pull of its planet(s) (e.g. Perryman 2000 for a review). The existence of one of these planets was confirmed independently when the partial occultation of the star by its planet was observed from the ground (Charbonneau et al. 1999), then from space (Brown et al. 2001; Vidal-Madjar et al. 2003). A number of groups are looking for planets using various techniques from the ground, but owing to the disturbing effects of the turbulent atmosphere, all exoplanets discovered so far are giant gaseous planets whose masses are comparable to that of Jupiter or Saturn. The main hope to collect a significant sample of Earth to Uranus-class planets in the coming years is to go to space. This is precisely one of the two main goals of the space mission COROT to be launched in 2005.

In this paper, we are concerned with the instrumentation, data analysis and expected performances of COROT in terms of exoplanet detection. In Sect. 2, we present the mission and describe the characteristics of the instrumental set-up. In Sect. 3, we review the detection method as it has been implemented in our simulations in order to compute the number of the expected detections. We discuss in Sect. 4 the detection efficiency of COROT as a function of the parent star magnitude and spectral type, and in Sect. 5 some effects that decrease the detection efficiency or may cause spurious detections.

Send offprint requests to: $\mathrm{P}$. Bordé, e-mail: Pascal.Borde@obspm.fr

\section{The mission and its instrumental set-up}

COROT was selected within the frame of the Small Mission Program of the French space agency CNES. It will cost typically 63 Meuros. Partners of CNES are several French laboratories: LAM (Marseille), LESIA (Meudon), IAS (Orsay), and several European countries: Austria, Spain, Belgium, and ESA/ESTEC. The goal of COROT is to perform high accuracy photometry on a total field as wide as $7.0 \mathrm{deg}^{2}$ in order to fulfil the requirements of the two main scientific objectives of the mission: a) measurement of stellar pulsations on a limited set of stars (Baglin et al. 1998) and b) detection of exoplanets transiting in front of their parent star (Rouan et al. 2000). COROT, the first transit mission in space, will have at least two followers: Kepler (Koch et al. 1998) on the American side, and Eddington (Favata et al. 2000) on the European side.

The two programs share the same instrument, featuring a $27 \mathrm{~cm}$ telescope without obscuration that includes two off-axis parabolas and a dioptric objective. Detection is achieved thanks to four cooled $\left(-40^{\circ} \mathrm{C}\right) \mathrm{CCD} 2048 \times 2048$ from $\mathrm{EEV}$, two devices being dedicated to each program. The CCDs are arranged according to an almost square pattern. The field of view of the exoplanets program is $3.5 \mathrm{deg}^{2}$. The orbit of COROT is pseudopolar, quasi-inertial, at an altitude of $\approx 900 \mathrm{~km}$. The generic platform, PROTEUS, provides a pointing stability of $\approx 0.2^{\prime \prime}$ when the instrumental information on the star positions is used. During the 2.5 years mission, five fields will be observed continuously, each one during 150 days. In order to prevent the limb of the Earth from being a source of background, COROT 
will be pointing in a direction not far from the equatorial plane. Since the Sun should also be avoided, the five fields are grouped around two main directions: one close to the Galactic Center $\left(l_{\mathrm{II}}=35^{\circ}, b_{\mathrm{II}}=0^{\circ}\right)$ and one close to the Anticenter $\left(l_{\mathrm{II}}=210^{\circ}\right.$, $\left.b_{\mathrm{II}}=0^{\circ}\right)$. After six months of observation in one of those directions, the satellite is rotated by $180^{\circ}$ with respect to the polar axis. The five fields will therefore be either 3 in the Center direction and 2 in the Anticenter direction or the reverse. The decision will be taken according to the launching date.

The goal of the exoplanet program will be achieved by monitoring continuously up to $12000 \mathrm{dwarf}$ stars in each field, with visual magnitudes from $V=11$ to $V=16$. The fields have been chosen at rather low galactic latitudes $\left(b_{\mathrm{II}}<10^{\circ}\right)$ in order to have a large density of stars. The technique used is aperture photometry: the flux collected during an elementary exposure of $32 \mathrm{~s}$ is measured by summing all pixels in a fixed mask encompassing the star Point Spread Function (PSF). The PSF covers $100-105$ px at $V=11-12,80-90$ px at $V=$ $13-14,40-50 \mathrm{px}$ at $V \geq 15$, and is itself a very low resolution on-axis spectrum of the star formed by a small biprism inserted a few centimeters above the exoplanet CCDs. For $V \leq 14$, this additional device provides color information in three bands (three subsets of the PSF pixels) that will be used as a powerful diagnostic tool to analyze doubtful events and may help to remove some of the stellar variability noise.

In order to cope with the data transmission rate of 1500 Mbits per day, data will be co-added on-board during periods of 8.5 min (16 exposures), before being downloaded. The integration will also be synchronized on the orbital period, so that any perturbation at the orbital frequency, such as the thermal fluctuations generated by Sun eclipses along the orbit, could be cancelled out at first order by summing the whole set of data taken during one orbital period. Time sampling will be improved, down to $32 \mathrm{~s}$ (one exposure) for any star where a high signal-to-noise event, like the transit of a giant planet, will be detected. This will make it possible to measure short-duration effects, such as limb-darkening on the stellar disk or variations of the transit period due to satellites around the planet or to other (non-occulting) planets (Sartoretti $\&$ Schneider 1998). Scattered light from the Earth limb is maintained at a low level thanks to the afocal telescope design and to a long baffle. The largest contribution among all sources of background originates from the zodiacal light that was evaluated to be no more than $16 \mathrm{e}^{-} \mathrm{s}^{-1} \mathrm{px}^{-1}$, using data from James et al. (1997).

\section{Detection method}

Exoplanet detection by the way of transit observations has been described many times in the literature (Rosenblatt 1971; Borucki \& Summers 1984; Jenkins et al. 1996). Let us remind the reader that at least three identical and equally spaced dimmings in a stellar light curve are interpreted as the signature of an occulting planetary companion. As the dimming relative depth is in the ratio of the surface of the planet to that of the star, it gives a measure of the planetary radius. In a previous paper (Bordé et al. 2001) we have used a simple cross-correlation treatment, equivalent to the matched filter approach of
Jenkins et al. (1996), to obtain a preliminary evaluation of the mission performances. Here, we will only review the key points of this method.

For the purpose of pure detection, especially at low $S / N$ (signal-to-noise ratio), the transit signal can be described by 4 parameters: the amplitude $A$ and duration $\tau$ of an individual transit, the transit period $P$ (equal to the revolution period of the planet), and the phase of the first transit $\phi$ (i.e. its date expressed as a fraction of $P$ ). The following method explores the $(\tau, P, \phi)$ parameter space by evaluating the likelihood of every triplet and gives the amplitude $A$ as a by-product. A tested triplet is referred to as a trial triplet.

For COROT targets, $\tau$ will be of the order of a few hours, certainly remaining below $\tau_{\max }=15 \mathrm{~h}$. Thus, the first step in the data processing will be to high-pass filter the light curves with a cut-off frequency of say $1 /\left(4 \tau_{\max }\right)$, in order to remove the irrelevant long-term stellar variations. The light curves are then averaged on a trial transit duration $\tau$ to increase the $S / N$, and cross-correlated with a transit-like signal at a trial period $P$. This noise-free signal has the shape of a comb with $k$ teeth, $k$ being the number of transits with a pe$\operatorname{riod} P$ during a 150 -day exposure on a given stellar field. Crosscorrelation products $C(\tau, P, \phi)$ have to be computed for enough trial triplets to correctly explore the parameter space. Detection occurs if a threshold fixed by a given confidence level is exceeded: $C \geq \beta \sigma_{C}$, where $\sigma_{C}$ is the standard deviation of the noise affecting $C$. We find that our requirement of less than one false detection for the entire mission is met for $\beta=7$, if a Gaussian statistics is assumed for $C$. This value is conservative as our parameter grid was not built to avoid correlated values of $C$. Indeed, our goal here is not to have a refined data processing as discussed recently by Jenkins et al. (2002) but instead to reach a first order estimate of the detection capability of COROT.

The minimum $S / N$ on a single transit necessary to assess a detection can be deduced from the above cross-correlation criterion:

$\frac{S}{N} \geq \frac{\beta}{\sqrt{k}}$

In turn, Eq. (1) leads to the minimum radius the planet should have to be detected:

$R_{\mathrm{P}} \geq R_{\star}\left(\frac{\beta}{\sqrt{k}} \frac{\sigma_{\mathrm{n}}}{N_{\mathrm{e}}}\right)^{\frac{1}{2}}$,

where $R_{\star}$ is the radius of the star, $\sigma_{\mathrm{n}}$ the standard deviation of the noise affecting the light curve and $N_{\mathrm{e}}$ the number of photoelectrons collected during $\tau$. In our simulations, we include the quantum noise, the read-out noise, the background noise (zodiacal light), the jitter noise and the stellar variability. The jitter noise, i.e. the photometric variations caused by the wobble of the PSF with respect to its photometric mask due to satellite pointing errors, will be corrected afterwards using the data from the seismology field. It amounts to $\approx 40 \%$ of the quantum noise. The stellar variability is assumed to be identical to that of the Sun and estimated to be $\simeq 50 \mathrm{ppm}$ rms at our timescale of interest using the SOHO data (Fröhlich et al. 1997). 


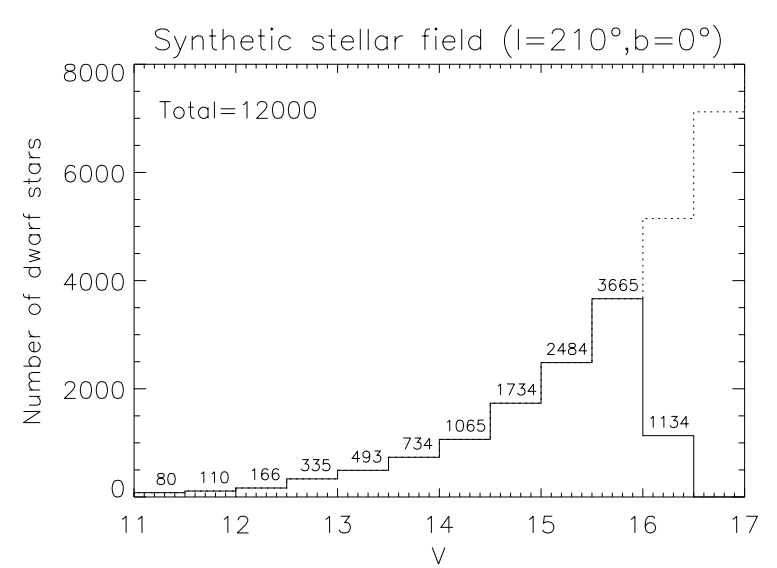

Fig. 1. Distribution of dwarfs in the $3.5 \mathrm{deg}^{2}$ synthesized field vs. the visual magnitude. Dashed line: complete Besançon model (16000 stars for $V \leq 16.5$ ). Solid line: selection of the 12000 brightest targets for COROT.

\section{Expected detections}

\subsection{Synthetic stellar field}

In order to assess the performance of COROT, we need realistic distributions of stars as a function of their visual magnitude $V$ and their spectral type $\mathrm{Sp}$ in the directions monitored by the satellite. Such distributions were synthesized with the stellar model of the Milky Way developed by Robin \& Crézé (1986, regularly updated), and retrieved from Besançon Observatory through their web interface. The $3.5 \mathrm{deg}^{2}$ target field for the simulations in this paper is centered in the direction of the Galactic Anticenter $\left(l_{\mathrm{II}}=210^{\circ}, b_{\mathrm{II}}=\right.$ $0^{\circ}$ ). It contains $\approx 16000 \mathrm{dwarfs}$ with magnitudes between 11 and 16.5, and spectral types between B0 and M5. The resolution used is 0.5 in magnitude, e.g. $V=14$ corresponds to $13.75 \leq V \leq 14.25$, and every spectral type is divided into 4 subclasses, e.g. G1 corresponds to $\mathrm{G} 0 \leq \mathrm{Sp} \leq \mathrm{G} 2$, G3 to $\mathrm{G} 2 \leq \mathrm{Sp} \leq \mathrm{G} 4$, and so on. The number of dwarfs as a function of $V$ roughly follows a geometric progression with a ratio of 2.0-2.5 (Fig. 1) and the whole sample peaks at spectral types F5-G0 (Fig. 2). Owing to the capacities of on-board electronics and telemetry of COROT, the total number of targets will be limited to $\approx 12000$ stars.

In this paper, we present only simulations regarding the Galactic Anticenter for which the Besançon model (with $A_{V}=$ $0.7 \mathrm{mag} / \mathrm{kpc}$ ) seems to agree well with preliminary stellar counts in actual fields. Thus, for the purpose of this work, we assume that COROT will observe 5 fields with statistical properties identical to those of this field. In the remainder of the paper, all figures regarding detection numbers are given for the entire mission: 5 fields, 150 days each, 60000 dwarfs in total.

Although stars of other luminosity classes (giants, subgiants...) are also present in the actual fields, they are not taken into account for the prospect of planet detection, since their large radii would lead to too weak transit signals. However, they contribute to the crowding effect and may induce an additional variability discussed in Sect. 5.2. When dealing with the observed fields, stars will be selected according to their luminosity classes, thanks to either specific ground

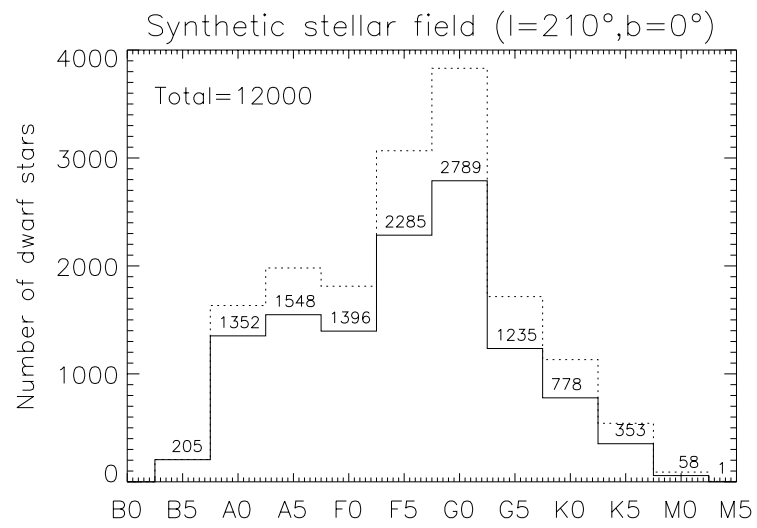

Fig. 2. Same as Fig. 1 vs. the spectral type.

observations (BVRI photometry) or the use of DENIS and 2MASS catalogues. For example, the DENIS survey (e.g. Epchtein et al. 1999) will provide the infrared photometry in the $I, J$ and $K$ bands down to magnitudes $I=18.5, J=16.5$ and $K=13.5$. Most giant stars in COROT fields could then be identified through the computations of their infrared colors (Epchtein et al. 1997), the others by way of dedicated observations if necessary. The COROT entry catalogue is currently being implemented by the Laboratoire d'Astrophysique de Marseille (LAM).

\subsection{Detections at a given orbital distance}

Since at least three transits are needed, we can compute the maximum distance at which a detection can occur as a function of the spectral type of the parent star. In the prospect of exobiology that is concerned with habitable zones around stars (e.g. Kasting et al. 1993; Franck et al. 2000), let us introduce the reduced orbital radius defined by $a_{\mathrm{r}}=a\left(L_{\star} / L_{\odot}\right)^{-0.5}$, so that a planet at $a_{\mathrm{r}}=1 \mathrm{AU}$ would receive as much flux from its star as the Earth from the Sun. Equivalently, one can use its effective blackbody temperature $T_{\mathrm{P}}(\mathrm{K})=278 / \sqrt{a_{\mathrm{r}}}$ (equal to the ground temperature only if the albedo is zero and without any greenhouse effect). As for a 150 day observation the revolution period $P$ should be less than 75 days (maximum value for 3 transits and $\phi=0$ ), we draw the accessible range of reduced orbital radii reported in Table 1. Based on the simulations by Franck et al. (2000), we conclude that depending on the age of the star, COROT could detect planets in the habitable zone around late $K$ to early M dwarfs, if such planets exist.

Now, let us assume that every dwarf in the synthetic field is orbited by one planet. The number of detected planets by COROT depends on the radii and (reduced) orbital distances of these planets, of the magnitude and spectral type of their parent stars and of the probability that at least three transits can be observed during a 150-day run. As a first hypothesis, we assume that all planets have the same radius $R_{\mathrm{P}}$ and are positioned at the same reduced orbital distance $a_{\mathrm{r}}$. Then, the number of detections reads $n_{\mathrm{det}}=n_{\star} \times p_{\mathrm{g}} \times p_{3}$, where $n_{\star}$ is the number of stars for which the criterion given by Eq. (2) is met, $p_{\mathrm{g}}$ the geometrical probability that transits are observable and $p_{3}$ the probability to observe at least 3 of them. Figures 3 and 4 display 
Table 1. Accessible range of reduced orbital distance/planet effective temperature as a function of the spectral type of the parent star.

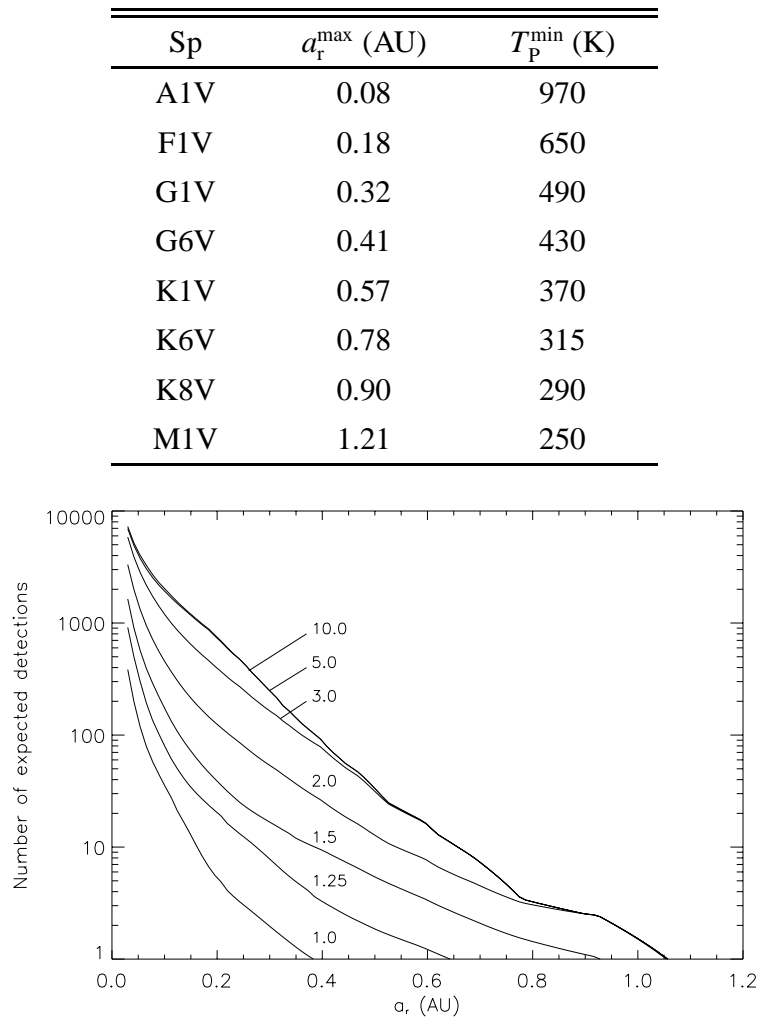

Fig. 3. Number of expected detections for the entire mission as a function of the reduced orbital distance for various planetary radii (expressed in unit of the Earth radius). It is assumed that every star has one planet of the labelled radius positioned at the considered distance.

detection curves, parameterized by the planet radius, as a function of $a_{\mathrm{r}}$ or $T_{\mathrm{P}}$. Selected results are also reported in Table 2. The ripples appearing for high $a_{\mathrm{r}}$ on Fig. 3 or low $T_{\mathrm{P}}$ on Fig. 4 are an effect of the finite size of the spectral type bins, and of the requirement on a minimum of 3 transits.

It appears from these computations that terrestrial planets (1-2 $R_{\oplus}$ ) are within reach of COROT provided their effective temperatures are $\gtrsim 500 \mathrm{~K}$. As can be expected from Eq. (1), the closer the planet, the higher the number $k$ of transits and the amount of detections. Besides, we can tell from the almost superimposed 5 and $10 R_{\oplus}$ curves that COROT reaches its full discovery potential as soon as $R_{\mathrm{P}} \gtrsim 5 R_{\oplus}$.

\subsection{Integrated number of detections}

As a second hypothesis, we estimate the expected detections by integrating along the $a_{\mathrm{r}}$ coordinate. This can be done provided an orbital distribution of planets around their parent stars is assumed. We have considered a uniform orbital distribution law: the planet probability density as a function of $a_{\mathrm{r}}$ is constant beyond $a_{\mathrm{r}}=a_{\mathrm{r}}^{\mathrm{min}}$ and normalized to one planet per star per $A U$ (arbitrary). To date, the actual observed value for $a_{\mathrm{r}}^{\min }$ is $0.03 \mathrm{AU}$ (Schneider 2002). This is consistent with the simulations of planet migration by Trilling et al. (1998). As the detection efficiency increases rapidly with the proximity of the

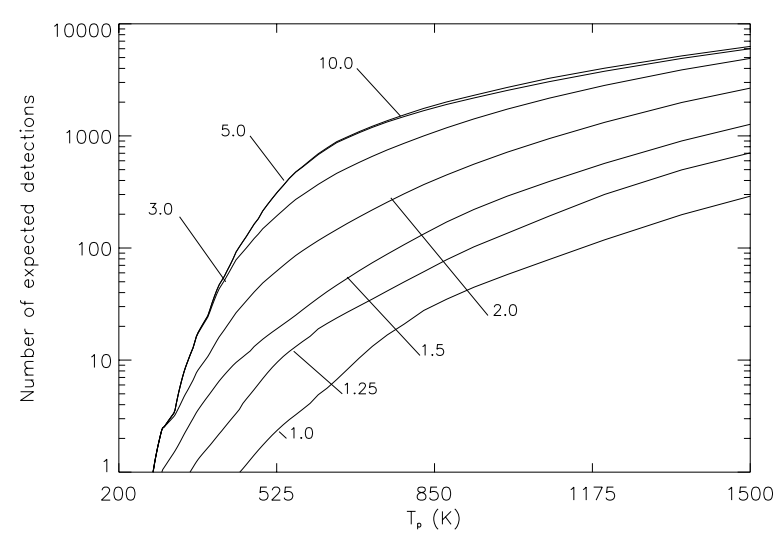

Fig. 4. Number of expected detections for the entire mission as a function of the planet effective temperature for various planetary radii (expressed in unit of the Earth radius). It is assumed that every star has one planet of the labelled radius with the considered effective temperature.

Table 2. Selection of expected detections for various planetary radii and reduced distances to the parent star/planet effective temperatures, assuming every star has one planet for such $a_{\mathrm{r}}$ and $R_{\mathrm{P}}$. Figures are given for the entire mission and a confidence of less than one false detection. The crowding effect (Sect. 5.2) is expected to remove $\approx 10 \%$ from the detections quoted here.

\begin{tabular}{ccrrrrr}
\hline \hline$a_{\mathrm{r}}(\mathrm{AU})$ & $T_{\mathrm{P}}(\mathrm{K})$ & $1 R_{\oplus}$ & $1.5 R_{\oplus}$ & $2 R_{\oplus}$ & $3 R_{\oplus}$ & $5 R_{\oplus}$ \\
\hline 0.05 & 1200 & 120 & 570 & 1320 & 2800 & 3800 \\
0.14 & 750 & 17 & 90 & 260 & 750 & 1300 \\
0.30 & 500 & 2 & 17 & 55 & 160 & 240 \\
0.86 & 300 & 0 & 1 & 3 & 3 & 3 \\
1.00 & 278 & 0 & 1 & 2 & 2 & 2 \\
\hline
\end{tabular}

planet to its star (Fig. 3), the integrated number of detections is very sensitive to $a_{\mathrm{r}}^{\min }$. In Table 3, we give the results for $a_{\mathrm{r}}^{\min }=0.03$ and $0.05 \mathrm{AU}$. At this stage, it is very important to recall that these figures hold for one planet per star. For giant planets around $\mathrm{G}$ stars, radial velocity observations have shown that this assumption largely overestimates the actual number of planets. Considering that for giant planets $\left(R_{\mathrm{P}} \simeq 10 R_{\oplus}\right)$ the radial velocity $(\mathrm{RV})$ detections are complete in the range 0 $0.1 \mathrm{AU}, \approx 18$ planets (Schneider 2002) have been detected out of $\approx 2600$ stars (Table 1 in Tabachnik \& Tremaine 2002). For a uniform orbital distribution, this translates into a frequency of $\approx 7 \%$ in the range $0-1 \mathrm{AU}$ and leads for COROT to $\approx 25 \mathrm{de}$ tections. However, because of the high level of confidence that is chosen, these figures should constitute the minimum that can be expected from COROT. Inversely, the comparison between these predictions and the actual number of planets detected by COROT will inform us about the frequency of these planets.

\subsection{Spectral type and magnitude of the parent star}

Up to this point, all the results drawn from our stellar sample were integrated on the spectral type $\mathrm{Sp}$ and the magnitude $V$ of the parent star. Our concern is now to analyze the influence of these two parameters on the detection capability of 

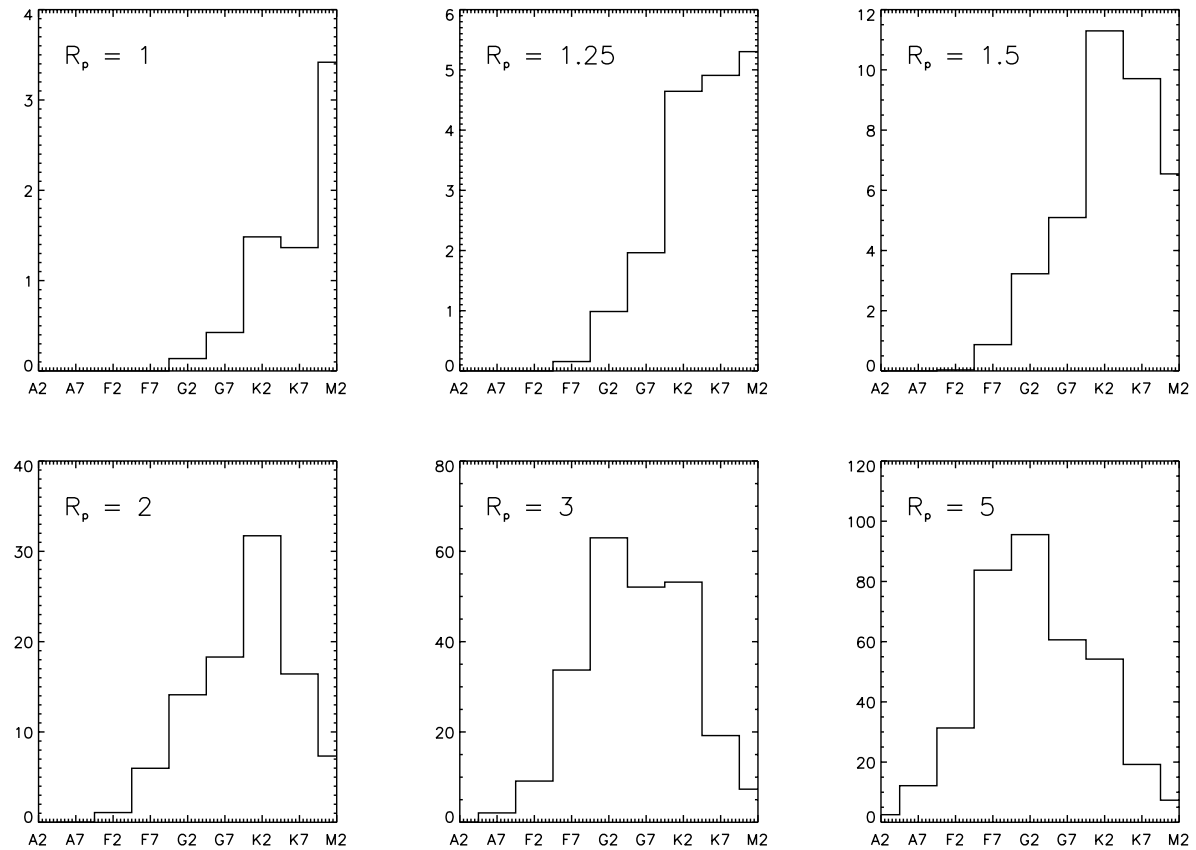

Fig. 5. Histograms of expected detections for the whole mission - with a false alarm rate less than one false detection - vs. the parent star spectral type, for various planetary radii (expressed in unit of the Earth radius). The planets are assumed to be uniformly distributed as a function of their reduced orbital distances. The crowding effect (Sect. 5.2) is expected to remove $\approx 10 \%$ from the detections plotted here.
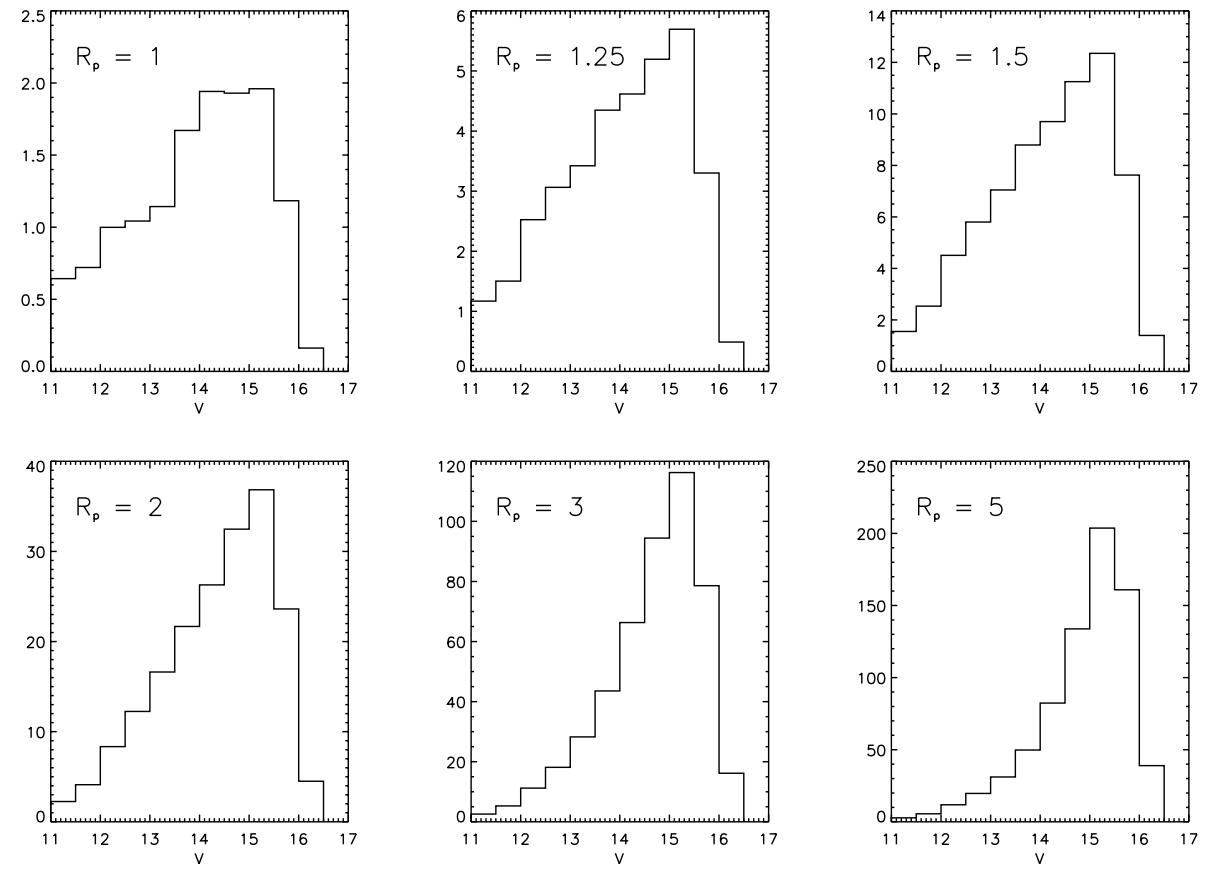

Fig. 6. Same as Fig. 5 vs. the visual magnitude of the parent star.

COROT. We have computed histograms of the number of detections vs. Sp and $V$ for different planetary radii (Figs. 5-6). Histograms for $R_{\mathrm{P}}=10 R_{\oplus}$ are not significantly different from those for $R_{\mathrm{P}}=5 R_{\oplus}$ and are not reproduced here. With respect to the spectral type, we note that the detection peak shifts progressively from $\mathrm{M} 2$ to $\mathrm{K} 2$ for terrestrial planets $\left(R_{\mathrm{P}} \lesssim 2 R_{\oplus}\right)$, then to $\mathrm{G} 2$ for Uranus-class objects $\left(R_{\mathrm{P}} \simeq 3 R_{\oplus}\right)$ and F7-G2 for giant planets $\left(R_{\mathrm{P}} \gtrsim 3 R_{\oplus}\right)$. This last feature can be attributed to the properties of the stellar sample itself that peaks around F5-G0. The histograms vs. $V$ show a general shape that strongly reflects that of the original stellar distribution (Fig. 1). As one goes deeper in magnitude, the number of detections keeps increasing because on one hand there are more stars, and on the other hand the detected planets are essentially hot objects frequently transiting in front of their parent stars. Therefore, we conclude that, for pure detection purposes, COROT targets should primarily be chosen among dwarfs later than F2 with magnitudes up to 16.5. This would lead to a slight improvement of the numbers given in Table 3 . 
Table 3. Integrated number of detections for the entire mission as a function of the planetary radius (the confidence level corresponds to less than one false detection). The higher value corresponds to $a_{\mathrm{r}}^{\min }=$ $0.03 \mathrm{AU}$ and the lower to $a_{\mathrm{r}}^{\min }=0.05 \mathrm{AU}$. The crowding effect (Sect. 5.2) is expected to remove $\approx 10 \%$ from the detections quoted here. The value for $10 R_{\oplus}$ is given for completeness as the frequency of such planets is known to be much less than 1 planet per star per reduced AU.

\begin{tabular}{rr}
\hline \hline$R_{\mathrm{P}}$ & $\begin{array}{r}\text { Integrated number } \\
\text { of detections }\end{array}$ \\
$\left(\right.$ in $\left.R_{\oplus}\right)$ & $5-6$ \\
1.0 & $12-18$ \\
1.25 & $26-37$ \\
1.5 & $70-95$ \\
2.0 & $189-240$ \\
3.0 & $300-367$ \\
5.0 & $(311-382)$ \\
\hline
\end{tabular}

Table 4. Impact of the stellar variability on the integrated number of detections expected for COROT. At the timescale of transits, the solar variability as estimated from $\mathrm{SOHO}$ data is $\simeq 50 \mathrm{ppm} \mathrm{rms}$. The crowding effect (Sect. 5.2) is expected to remove $\approx 10 \%$ from the detections quoted here.

\begin{tabular}{rrrr}
\hline \hline$R_{\mathrm{P}}$ & solar var. & solar var. & solar var. \\
(in $\left.R_{\oplus}\right)$ & $\times 1$ & $\times 10$ & $\times 50$ \\
\hline 1.0 & 6 & 2 & 0 \\
1.25 & 18 & 4 & 0 \\
1.5 & 37 & 10 & 0 \\
2.0 & 95 & 32 & 2 \\
3.0 & 240 & 112 & 12 \\
5.0 & 367 & 289 & 73 \\
10.0 & 382 & 377 & 274 \\
\hline
\end{tabular}

\section{Effects decreasing the detection efficiency}

\subsection{Stellar variability}

It is recognized that compared to other dwarfs, the Sun is a pretty quiet star. Since the Sun has been chosen as the prototype for our model of stellar variability, one may wonder what would happen if this noise source were moderately or even considerably higher. We have investigated the consequences of such an increased variability on the integrated number of detections (Sect. 4.3) by computing the number of detections for a stellar variability 10 and 50 times higher than the solar value (Table 4$)$. It is found that a high variability $(\times 10)$ causes a loss of terrestrial planets by a factor 3-4, whereas a very high variability $(\times 50)$ makes their detection out of reach. In that case however, we consider taking advantage of the color information contained in the PSF (Sect. 2) to remove part of this noise with a proper combination of the colored channels (Bordé et al. 2003).

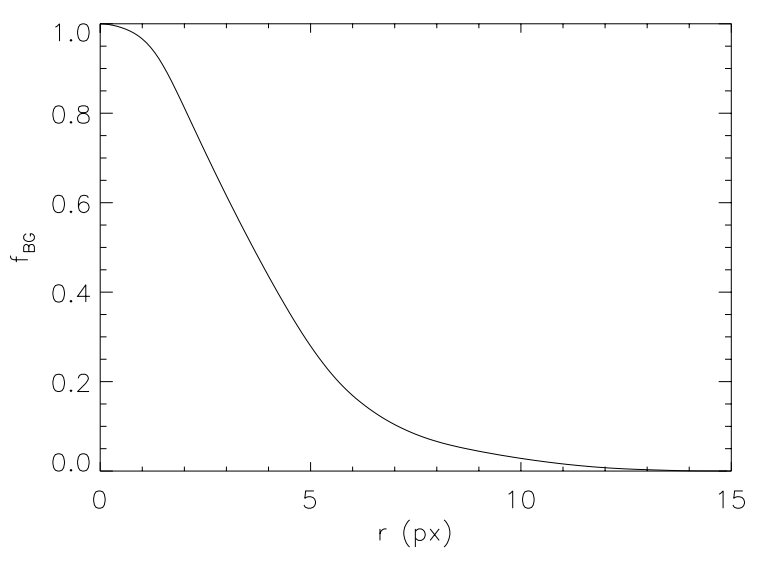

Fig. 7. Fraction of a background star flux included in a target mask vs. the distance between their photocenters, once averaged over all relative orientations.

\subsection{Background induced variability}

In most stellar fields close to the Galactic Plane, the number of background (BG) stars increases as one goes deeper, typically by a factor 2.3 per magnitude. Consequently, in any target star mask, there will be some flux contribution by BG stars, some of which are variable. This will add to the photometric measurement an extra noise with a time dependence similar to that of the target star variability. In this section, we derive a rough estimate of the fraction of the detections that are lost because of this extra noise.

Variability of dwarf and giant stars has been measured by the Geneva group and the Hipparcos mission (Grenon 1993; Eyer \& Grenon 1997). Crudely, the result can be described as:

- no detected variability for $90 \%$ of dwarfs;

- $10 \%$ of dwarfs have $1.4 \times 10^{-2}$ variability rms;

- $98 \%$ of giants have, at most, $2 \times 10^{-3}$ variability rms;

$-2 \%$ of giants have, at most, $5 \times 10^{-2}$ variability rms.

These variabilities have been measured on timescales of one to few months whereas transit search by COROT is mostly concerned with timescales of 15 hours or less. In the absence of information at proper timescales, the latter is derived from the former using the frequency power spectral density of the solar variability (DIARAD/SOHO, Batalha 2001). Fortunately, this scaling is not very sensitive to the exact frequencies selected. Typically, a 0.16 scaling factor is found from longer (months) to shorter (hours) timescales.

The fraction $f_{\mathrm{BG}}$ of the BG star flux included in the target mask depends on the relative orientations of the target mask and the BG star PSF, and on the distance $r$ between their photocenters. Once averaged over all relative orientations, $f_{\mathrm{BG}}$ is a decreasing function with half maximum at $r=3.6 \mathrm{px}$ for a 85 px mask (Fig. 7). A Monte-Carlo simulation is performed for every bin of the target star magnitude $V$, using the BG stellar densities provided by the Besançon model and the above variability laws. Thus, we compute histograms of the number of target stars affected by variable BG stars vs. the standard deviation $\sigma_{\mathrm{BG}}$ of this extra variability noise. This effect is considered as perturbing significantly the detection if it 
increases the standard deviation of the total noise by $20 \%$, i.e. $\sigma_{\mathrm{n}}^{\prime}=1.2 \sigma_{\mathrm{n}}$ or $\sigma_{\mathrm{BG}}=0.66 \sigma_{\mathrm{n}}$ as all sources of noise are uncorrelated. For instance, the extra variability would prevent the detection of a planet with a radius $R_{\mathrm{P}}=1.5 R_{\oplus}$ orbiting a K2 star at $a_{\mathrm{r}}=0.05 \mathrm{AU}$ if $\sigma_{\mathrm{BG}}>1.02\left(R_{\mathrm{P}} / R_{\star}\right)^{2} N_{\mathrm{e}}$ (Eq. (2)).

For every bin of magnitude, the number of target stars for which BG stars induce such a level of extra noise is deduced from the histograms of the Monte-Carlo simulation. The fraction of lost detections is obtained by dividing the number of polluted targets by their total number. Then a weighted average is calculated over the different bins, with the expected number of detections as a weight. The loss amounts to $13 \%$ for $R_{\mathrm{P}}=1.5 R_{\oplus}$ and $10 \%$ for $R_{\mathrm{P}}=3 R_{\oplus}$. This fraction seems to vary slowly with the mask size: for a smaller mask with $60 \mathrm{px}$, the loss becomes respectively $11 \%$ and $8.5 \%$. For mask sizes to be used by COROT, it is approximatively proportional to the square root of the number of pixels in the mask.

As a conclusion, the extra variability induced by BG stars is a systematic effect that causes a loss of $\approx 10 \%$ of the detections. This impact, although limited, is not negligible.

\subsection{Background eclipsing binaries}

Most of the time, eclipsing binaries are readily distinguished from transiting planets by the fact that they lead to two transits of unequal depth, both lasting longer than expected for a planet. Low amplitude grazing transits of (almost) identical stellar companions may be distinguished from a unique transiting planet with half the revolution period thanks to the characteristic V shape of grazing transits as opposed to a nearly flat shape for planets (e.g. Borucki et al. 2001). Low $S / N$ candidates for which this criterion could not be applied would be the subject of complementary spectroscopic observations for a definitive classification.

A tricky case happens when a partly overlapping BG star (but not the foreground one) is an eclipsing binary of identical components or features only primary transits because the companion is too faint. It may then mimic a planetary transit around the foreground star. If the magnitude of the foreground star is $V \leq 14$, then a color discrimination could be done: only if the superposition of both PSFs is almost perfect, spurious transits due to the BG star would not be present in all color channels. Cases where $V>14$ are more difficult. At the beginning of every 150 day observation of a given field, it is planned to make a long exposure (typically a couple of hours) to select the target stars and to define their corresponding aperture masks. This exposure will allow us to detect potential polluting BG stars. For every planet candidate orbiting a star potentially polluted, complementary spectroscopic observations would have to be conducted as well.

\section{Conclusion}

With these simulations, we have shown that COROT has the capacity to detect numerous exoplanets, not only Jupiter and Uranus-class ones, but also hot terrestrial planets, if they exist. Hot terrestrial planets should be mainly gathered around 14-15th magnitude K2-M2 stars, Uranus-class planets around 15th magnitude $\mathrm{G} 2$ stars and giant ones around 15-16th magnitude F7-G2 stars. The number of detections increases with the magnitude of the parent star up to $V=16$, thus reflecting the original stellar distribution. However, only hot and frequently transiting planets may be found around faint stars.

Besides, we have evaluated the impact of a high stellar variability noise, and of the crowding effect. If all star variabilities were to be orders of magnitude above the Sun level, (an unlikely situation), no terrestrial planet could be within reach (apart maybe from the use of the color information). Finally, detection estimates must take into account the crowding effect that may cause a loss of about $10 \%$ of the detections by inducing an extra variability noise.

As a final remark, let us point out that COROT will bring the first data about the abundances and the orbital distributions of Uranus-class to terrestrial exoplanets. These constitute key information for the ambitious followers like Darwin (Léger et al. 1996; Fridlund et al. 2000) and NASA's Terrestrial Planet Finder (Beichmann et al. 1999).

Acknowledgements. We are very grateful to Dr. Michel Grenon for valuable discussions on stellar variability, and to Dr. Marc Ollivier for correcting a few inaccuracies and for his help on taking into account the jitter noise.

\section{References}

Baglin, A., \& The COROT Team 1998, Asteroseismology from space - The COROT experiment. In New Eyes to See Inside the Sun and Stars, IAU, 185, 301

Batalha, N. 2001, in Stellar Structure and Habitable Planet Finding, Proc. of the 1st Eddington workshop, Córdoba, 11-15 june 2001, ed. F. Favata, I. W. Roxburgh, \& D. Galadí-Enríquez, ESA SP-485

Beichmann, C. A., Woolf, N. J., \& Lindensmith, C. A. 1999, TPF, Origins of stars, planets and life (Pasadena, CA: JPL publication), JPL99-3

Bordé, P., Rouan, D., \& Léger, A. 2001, C. R. Acad. Sci. Paris, série IV, 1

Bordé, P., Léger, A., Rouan, D., \& Cameron, A. C. 2003, A\&A, submitted

Borucki, W., \& Summers, A. 1984, Icarus, 58, 121

Borucki, W., Caldwell, D., Koch, D. G., et al. 2001, PASP, 113, 439

Brown, T. M., Charbonneau, D., Gilliland, R. L., Noyes, R. W., \& Burrows, A. 2001, ApJ, 552, 699

Charbonneau, D., Brown, T. M., Latham, D. W., \& Mayor, M. 1999, ApJ, 529, L45

Epchtein, N., de Batz, B., Capoani, L., et al. 1997, The Messenger, March, 27

Epchtein, N., Deul, E., Derriere, S., et al. 1999, A\&A, 349, 236

Eyer, L., \& Grenon, M. 1997, ESA Hipparcos Symp. at Venice, ESA SP-402, 467

Favata, F., Roxburgh, I., \& Christensen-Dalsgaard, J. 2000, Eddington, Assessment Study Report, ESA-SCI(2000)8

Franck, S., Block, A., von Bloh, W., et al. 2000, Planet Space Sci., 48, 1099

Fridlund, M., Volonte, S., Laurance, R., et al. 2000, Darwin infrared space interferometer, Concept and feasibility study report, ESASCI $(2000) 12$ 
Fröhlich, C., Andersen, B. N., Appourchaux, T., et al. 1997, Sol. Phys., 170,1

Grenon, M. 1993, in Low-level stellar variability, ed. W. Weiss, \& A. Baglin, ASP Conf. Ser., 40, 603

James, J. F., Mukai, T., Watanabe, T., Ishiguro, M., \& Nakamura, R. 1997, MNRAS, 288, 1022

Jenkins, J. M., Doyle, L. R., \& Cullers, D. K. 1996, Icarus, 119, 244

Jenkins, J. M., Caldwell, D. A., \& Borucki, W. J. 2002, ApJ, 564, 495

Kasting, J. F., Whitmire, D. P., \& Reynolds, R. T. 1993, Icarus, 101, 108

Koch, D., Borucki, W., Webster, L., et al. 1998, in Space Telescopes and Instruments V, ed. P. Y. Bely, \& J. B. Breckinridge, Proc. SPIE, 3356, 599

Léger, A., Mariotti, J.-M., Mennesson, B., et al. 1996, Icarus, 123, 249

Queloz, D. 2001, in Stellar Structure and Habitable Planet Finding, Proc. of the 1st Eddington workshop, Córdoba, 11-15 June 2001, ed. F. Favata, I. W. Roxburgh, \& D. Galadí-Enríquez, ESA SP-485
Perryman, M. A. C. 2000, Rep. Prog. Phys., 63, 1209

Robin, A., \& Crézé, M. 1986, A\&A, 157, 71

http://www. obs-besancon.fr/modele/modele.html

Rosenblatt, F. 1971, Icarus, 14, 71

Rouan, D., Baglin, A., Barge, P., et al. 2000, Detecting Earth-Uranus class planets with the space mission COROT. In Darwin and Astronomy - The Infrared Space Interferometer, ESA SP-451, 221

Sartoretti, P., \& Schneider, J. 1998, A\&AS, 134, 553

Schneider, J. 2002, The Extrasolar Planets Encyclopaedia, http://www.obspm.fr/planets/

Tabachnik, S., \& Tremaine, S. 2002, MNRAS, 335, 151

Trilling, D. E., Benz, W., Guillot, T., et al. 1998, ApJ, 500, 428

Vidal-Madjar, A., Lecavelier des Etangs, A., Désert, J.-M., et al. 2003, Nature, 422, 143 